\title{
Challenges and Strategy Design for the Globalization of Traditional Chinese Drugs
}

\author{
Haitao TANG $^{1}$ \\ Jimei $\mathrm{MA}^{2}$ (i) \\ Li LIU ${ }^{3}$ \\ Wenlong HUANG ${ }^{4}$
}

'School of International Pharmaceutical Business, China Pharmaceutical University, China; Jiangsu Suzhong Pharmaceutical Group Co., Ltd., China

'Email:mtang@vip.sina.com Tel:15261029666

Jiangsu Suzhong Pharmaceutical Group Co., Ltd., China

EEmail:mim0607@hotmail.com Tel:15896039811

${ }^{*}$ School of International Pharmaceutical Business, China Pharmaceutical University, China

${ }^{3}$ Email:cpuliuli123@163.com Tel:15298367937

Email:ydhuangwenlong@126.com Tel:(025)86636655

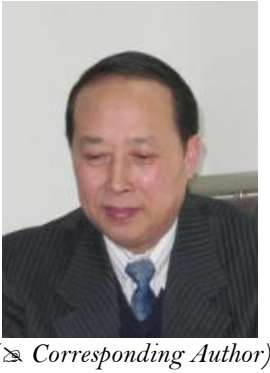

\begin{abstract}
As economic exchange has deepened in the international community, traditional Chinese drugs have gone global and gained a share in overseas markets. However, due to internal and external restrictions, only a small proportion of traditional Chinese drugs can gain access to mainstream drug markets in foreign countries. This article designs strategies and provides suggestions for globalizing the presence of traditional Chinese drugs based on an analysis of the current conditions and challenges of this globalization process.
\end{abstract}

Keywords: Globalization of traditional Chinese drugs, Challenges, Strategy design.

JEL Classification: F407.7.

Citation | Haitao TANG; Jimei MA; Li LIU; Wenlong HUANG (2018). Challenges and Strategy Design for the Globalization of Traditional Chinese Drugs. Asian Journal of Social Sciences and Management Studies, 5(1): 40-45.

History:

Received: 26 January 2018

Revised: 27 February 2018

Accepted: 2 March 2018

Published: 5 March 2018

Licensed: This work is licensed under a Creative Commons

Attribution 30 License (c)

Publisher:Asian Online Journal Publishing Group
Contribution/Acknowledgement: All authors contributed to the conception and design of the study.

Funding: This study received no specific financial support.

Competing Interests: The authors declare that they have no conflict of interests.

Transparency: The authors confirm that the manuscript is an honest, accurate, and transparent account of the study was reported; that no vital features of the study have been omitted; and that any discrepancies from the study as planned have been explained.

Ethical: This study follows all ethical practices during writing.

\section{Contents}

1. Internationalization Status of TCM 41

2. Existing Problems in the Internationalization of TCM

3. Design, Selection and Guarantee of TCM Internationalization Strategies 43

References..... 
As a traditional medical system that has endured for thousands of years in China, Traditional Chinese Medicine (TCM) is well preserved and extensively influential. TCM internationalization has a solid international background, and traditional medicine has attracted more and more attention from governments worldwide (Balunas and Kinghorn, 2005). The Europe Union (EU) Directive for Registration Procedure of Traditional Herbal Medicine and the United States (US) Guide to Herbal Medicine Products indicate that the EU and the US have already recognized herbal medicine as a type of medicine. Generally speaking, TCM currently has the scale and conditions to expand globally.

\section{Internationalization Status of TCM}

\subsection{Import and Export Status of TCM Products of China}

According to export data from 2013-2016, the import and export status of TCM products (including extracts, Chinese patent medicines, TCM materials, herbal medicine and slices for decoction; see Fig. 1 to Fig. 3). For these four years, the export amount of TCM products exported from China was 30 billion US dollars. From 2013 to 2015 , it displayed a trend of rising slowly. In 2016, it declined slightly, by $8.8 \%$, compared with 2015. In 2016, the import amount of TCM products grew by 13.7\%. Overall, the export amount of TCM products of China is far higher than the import amount, which reflects the advantages of the TCM materials in China. However, the analysis of the gap between imports and exports (export amount and import amount) reveals a trend of a modest increase from 2013 to 2015 , and a slight decline of $16.2 \%$ is observed in from 2015 to 2016.

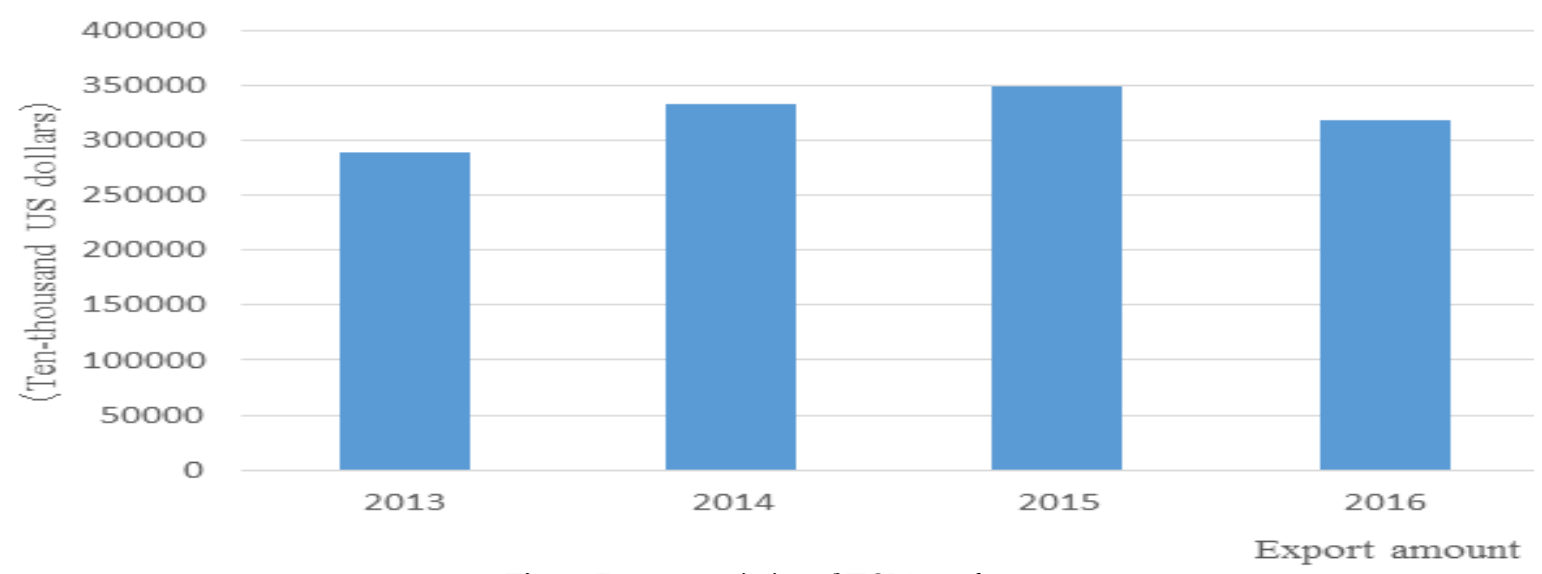

Fig-1. Export statistics of TCM products

Source: Statistical data of China Chamber of Commerce for Import\& Export of Medicines and Health Products

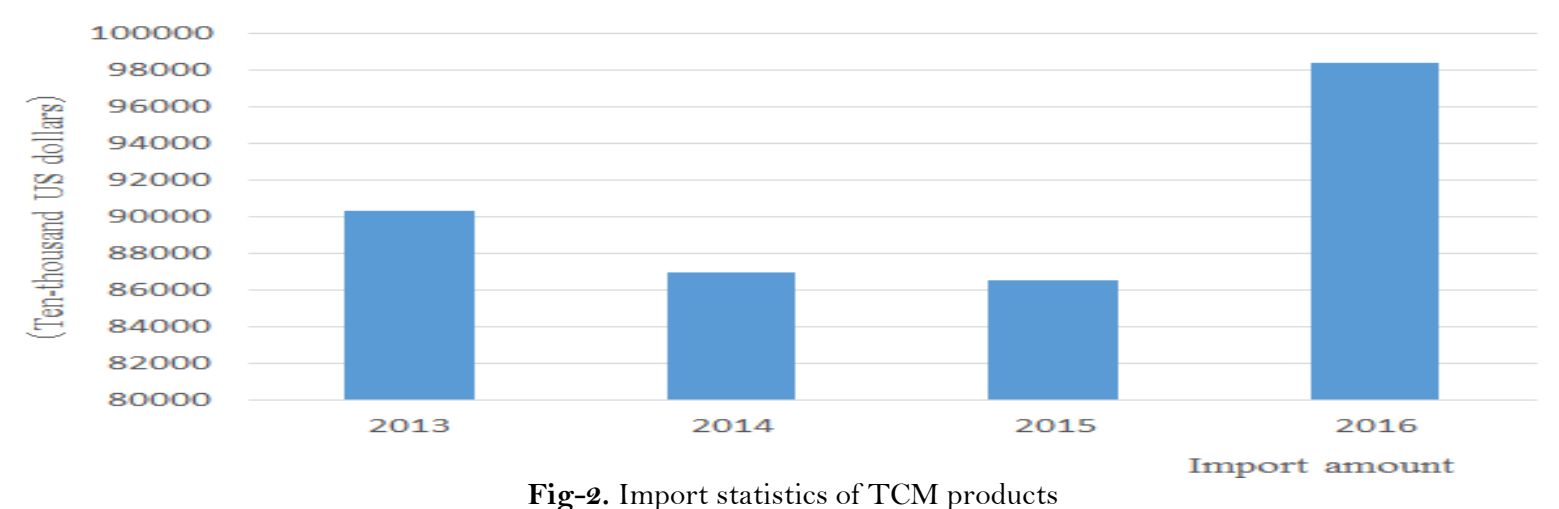

Source: Statistical data of China Chamber of Commerce for Import\& Export of Medicines and Health Products

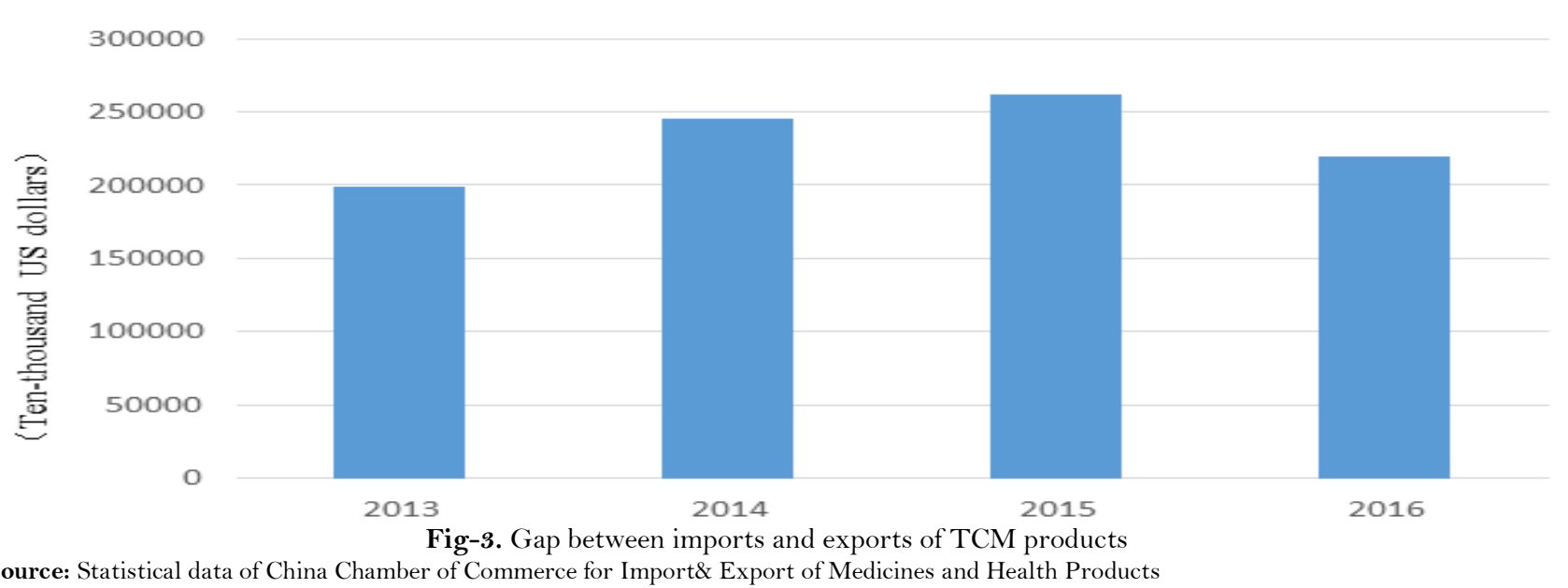

The literature and statistical data indicate that China mainly exports products such as extracts, herbal medicine and slices for decoction (Martins, 2014). The statistical data in Figs. 4 and 5 show that the export amount of Chinese patent medicine accounts for only $7 \%$, medicinal herbs and medicinal slices approximately $30 \%$, and exports $60 \%$. 


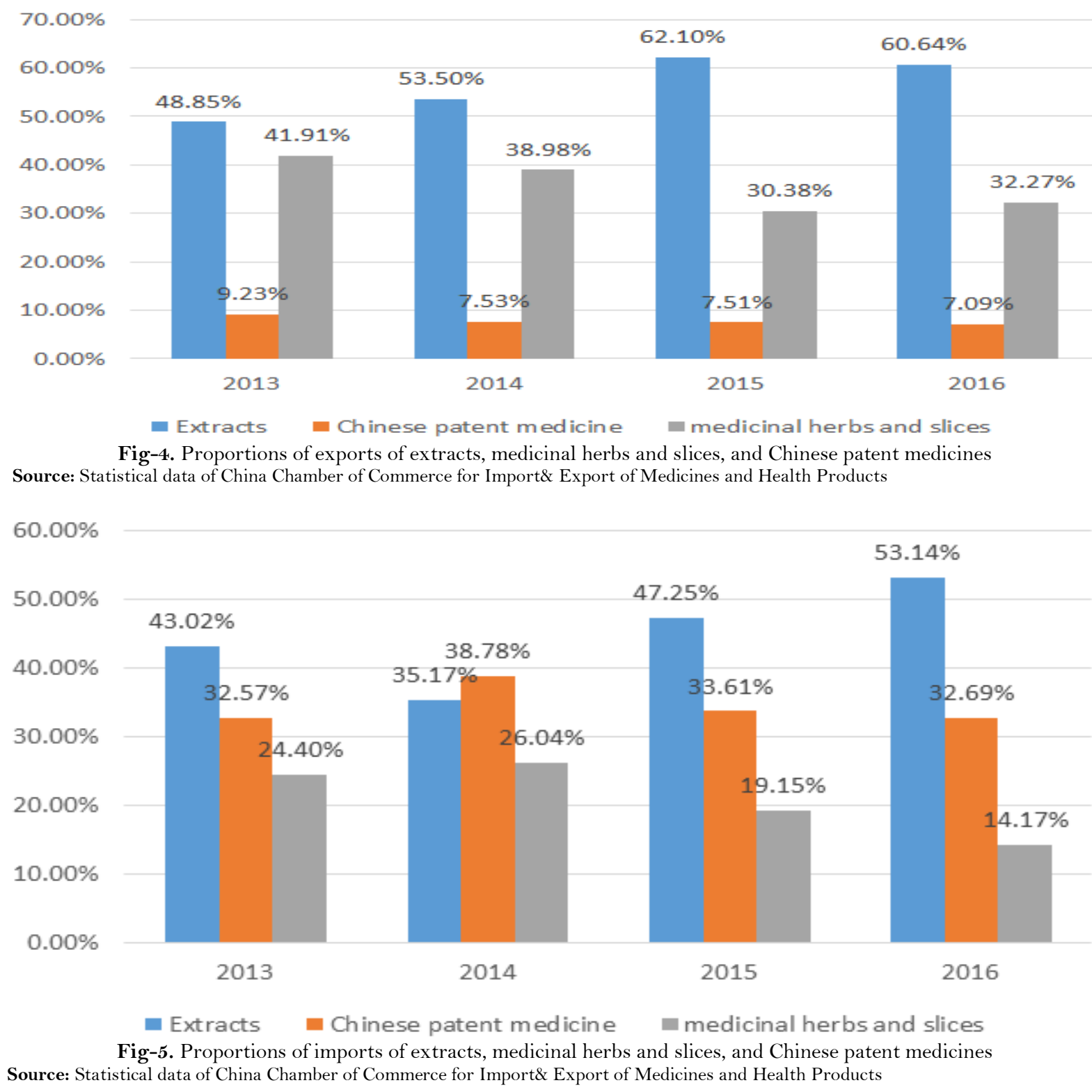

\subsection{Registration and Approval Status of TCM Abroad}

Although TCM has spread from China to many countries and regions, its share of the global market is still less than $5 \%$. Only a limited number of TCM products have entered the global mainstream medicine markets as medicine (Ya et al., 2011).

At present, nine kinds of TCM products from China have been registered with FDA, including Compound Danshen Dripping Pills, which have completed a stage III clinical test and witnessed the fastest internationalization; Xingling Granules and Kanglaite Injection, which are undergoing a stage III clinical test; and six other products, such as Xuezhikang, Guizhi Fuling Pills and Chuanxinlian Capsule, which are undergoing a stage II clinical test.

In the EU, Di'ao Xinxuekang and Danshen Tablets have been registered successfully in Holland. Danggui Concentrated Pills are applying for registration in Sweden. Ginkgo Leaf Tablets and Pill of Six Ingredients with Rehmannia are under registration in Holland.

\section{Existing Problems in the Internationalization of TCM}

According to a comprehensive analysis, the problems to be solved in the internationalization of TCM lie in the following seven areas.

\subsection{It is Difficult for TCM Culture to be recognized}

TCM and Western Medicine are different medical theoretical systems with different modes and substantial differences in such aspects as theoretical foundations, methods of thinking, and diagnostic and treatment approaches. In the latter, the treatment are partial; in the former, symptoms and root causes are treated simultaneously. TCM works slowly and requires a long course of treatment. Due to differences in cultures and languages, the concept of TCM has not been globally recognized and accepted (Van et al., 2010).

\subsection{Unclear Material Basis and Function Mechanism}

TCM contains complex contents, so it difficult to identify its composition. As a result, the effective contents of TCM are not clear. We cannot confirm the specific mechanisms and pharmacologic actions. Due to this uncertainty, quality control indicators may be irrational and even fail to display the relevance of the curative effects (Wagner et al., 2016). As a result, the medicine quality testing standards cannot correctly reflect the specific clinical effects of the medicine.

Medicine has long been used based on TCM theory and experience. Basic research on TCM has lagged behind, relatively speaking. There are no clear effective component contents or regular testing methods. No studies have 
been conducted in such areas as pharmacological toxicity, standardization or regulation, and research has not caught up with the international technological levels (Wagner et al., 2016).

\subsection{Singular Product Indicators Cannot Guarantee the Evenness and Effectiveness of Medicine}

Though the standards of TCM products in China have been enhanced to some extent, the product indicators are still singular, which makes it difficult for other countries to accept and recognize the curative effects of TCM. TCM contains many chemical components. The key components of some TCM may not be the effective components; the trace components may be the important curative effect components. Due to their herbal materials and the technologies for processing TCM, quality consistency across different batches of TCM may be relatively low (Wang et al., 2005). Some quality cannot be truly reflected by adopting one or several component indicators.

\subsection{Lacking Persuasive Clinical Data}

TCM clinical care in China still mostly stops at medical experience, but scientific clinical pharmacological data are lacking. Meanwhile, the basic matters of the curative effects of TCM have not been identified. It is difficult to identify the components that play a leading role in the medicine or to make a scientific interpretation of the treatment mechanism of such components (Normile, 2003) In recent years, China has just begun to formulate and implement standards and regulations, such as Good Clinical Practice and Good Laboratory Practice (GLP), which are still different from the globally prevailing standards. Therefore, in evaluating the pharmacology, curative effects and toxicity of TCM, the global community has not quite recognized related testing data.

\subsection{Planting of Crude Herbal Materials to be regulated}

In each link of herbal material planting, most TCM materials in China lack professional management and unified standards and testing methods. Medicinal herb planters still cannot control the quality of herbal materials. Therefore, excessive residue of pesticides, heavy metal content, and differing contents of effective components commonly occur. Generally, the heavy metal content indicator specified by foreign countries is stricter than the range of heavy metal content permitted in China (Liang et al., 2004). Therefore, the TCM products that are produced and tested following the standards of China will face obstacles in exportation.

\subsection{The Production Process Control is Extensive}

Though the TCM industry in China has developed rapidly in recent years and passed the domestic good manufacturing practice (GMP) certification, some enterprises have still lagged behind in the specific implementation of the production process management of medicines and cannot meet the requirements of the international market regarding the production quality of medicines. In TCM quality testing and control, most enterprises are still at the qualitative testing level and conduct quantitative tests on only some indicators (Martins, 2014). In addition, the quality control system is far from complete, without very strict requirements on such aspects of medicines as quality control, the monitoring of adverse reactions and GMP requirements.

\subsection{Intellectual Property Protection Issue}

Although the protection of intellectual property for TCM in China has been increasingly regular and legal, there are still many problems related to how to apply the protection. On one hand, enterprises have not paid enough attention to the protection of their intellectual property and have not made any corresponding attempts to protect their intellectual property. Since China has long lacked awareness of and systems for the protection of intellectual property for TCM, our scientific and technological fruits have gone unprotected. Many classical TCM prescriptions and other proven prescriptions have been utilized by foreign pharmacy companies for free.

On the other hand, the protection system for intellectual property of TCM is not complete enough. In contrast to Western Medicine, each batch of TCM is unique; it has a medicinal formula but not specific chemical structural formula. The adjustment of the dosage of any medicinal material in the formula may form a new formula. If there is any imitation, in judging whether there is infringement, all components and specific dosages of each component must be confirmed. Therefore, it is rather difficult to identify infringement, and infringement is very easy.

\section{Design, Selection and Guarantee of TCM Internationalization Strategies}

Certain problems hinder the internationalization process of TCM products. Therefore, this paper offers suggestions based on actual situations so that more enterprises can internationalize TCM smoothly.

\subsection{Cultural Strategy for National Competition: Promote the Spread and Communication of TCM Culture}

\subsubsection{Strengthening the International Communication on TCM Culture}

Developing TCM requires the publicity of TCM culture. To strengthen cultural exchange and reduce the factors that hinder the exchange between Eastern and Western cultures, we must constantly promote TCM culture, adopt scientific methods to exhibit the action mechanisms and cultural connotations of TCM and break the conventional thinking limitations of Western medicine theories.

\subsubsection{Promoting the International Demonstration Exchange and Cooperation of TCM}

We should make full use of related resources at home and strengthen the international exchange and cooperation related to TCM, thereby promoting the development of TCM culture. Cooperation and exchange include strengthening the policies and regulations for traditional medicine, herbal medicine quality standards and control indicators and the formulation of various management regulations. We should strengthen the exchange between the Chinese government, the leadership of international organizations and the cooperation with foreign institutions of higher learning and hospitals and co-establish clinical research centres or groups to make more academic authority institutions accept and recognize TCM. 


\subsection{Support Strategy for National Competition: Step Up the Governmental Support}

\subsubsection{Strengthening the Coordination Mechanism at the Government Level}

In the course of TCM internationalization, there are many qualifications, procedures and departments involved as well as strict requirements on the time and procedure of review and approval. The government should adopt corresponding encouraging policies, including support such as funds and technologies for export enterprises, policy support for domestic review and approval procedures, promoting qualified enterprises to carry out related work on TCM internationalization, and providing effective information consultation and suggestions for domestic enterprises to find the best routine and method for internationalization.

\subsubsection{Establishing a Compensation Mechanism for TCM Internationalization}

The government should pay attention to and support TCM enterprises. For example, appropriate policy support may be offered to enterprises that have passed the EU or American FDA certification and embarked on TCM internationalization. The internationalization process of TCM is long and costly, so if the government can offer financial support to smoothen this process, it will effectively enhance the international competitiveness of Chinese TCM enterprises and stimulate their activeness, thereby promoting the internationalization process of the whole TCM industry.

\subsection{Innovation Strategy: Increase Scientific Research Efforts in TCM}

\subsubsection{Increasing the Input into TCM Research}

It is an important foundation for TCM internationalization to enhance its international competitiveness and establish the value of its use. The fundamental reason why TCM encounters all sorts of difficulties in internationalization lies in the fact that TCM is a summary of clinical experience, and micro-studies on the medicine itself are lacking. The state and enterprises should increase investment in the studies on TCM, speed up the TCM research and the renewal and upgrade of production equipment, produce TCM products with a high added-value and meet the international requirements. Only in this way can TCM be competitive in the world.

\subsubsection{Referring to Foreign Natural Medicine Regulations, Doing Well in Structural Adjustment of Natural Medicines}

To align the foreign regulations concerning natural medicines, TCM can start with individual herbs. For example, enterprises can take some formula of TCM with specific curative effects and the effective part as an effective body, control the composition and contents of the "effective body", and gradually reach the purposes of "safety, control, stability and effectiveness". The "effective body" in individual herbs can also be analysed and summarized with the "assistant and guide" theory of TCM.

\subsubsection{Developing New Dosage Forms and Products for the Demands of the Global Market}

We should break the limits of the traditional TCM dosage forms of China and develop dosage forms and products that meet the demands of international market and are accepted by the consumers. The TCM industry should increase the input of manpower and materials, break the limits of conventional production methods, and renew and upgrade the production process towards the process of extraction and refinement in order to enhance the scale and level of the production process.

With advantages such as few toxic side effects and enduring curative effects, TCM is expected to make further breakthroughs in the treatment of cancer, Acquired Immune Deficiency Syndrome (AIDS), cardiovascular diseases and chronic diseases. Therefore, we should explore and study the traditional formulas and carry out secondary development with modern innovative technologies to develop and produce new products, which will play a huge role in spreading TCM into the global market.

\subsection{Standard Strategy: Enhancing the Standardization and Modernization of TCM Quality}

\subsubsection{Enhancing the Standardization of TCM Quality}

Regarding medicine standards, many countries have specified the limits of heavy metals and residues of pesticide of TCM and begun to enhance the standards. We should release TCM product quality standards that are applicable to both China and the world as soon as possible. Establishing an international standard system for TCM and unifying the TCM standards will also influence the global market to a certain extent, including strict management of planting and production, thereby guaranteeing that the consistency of TCM quality can be controlled.

\subsubsection{Enhancing the Modernization of TCM Quality}

TCM modernization is critical, and adopting advanced technologies is a necessary step in modernization. We should regulate each node of the TCM industry, apply high and new technologies, use advanced equipment and adopt strict standards to transform the TCM production and reach the goals of modernizing the technologies for the extraction, analysis and production of TCM based on related conventional theories.

\subsection{Talent Strategy: Cultivation of High-Quality TCM Talent}

\subsubsection{Striving to Cultivate Cross-Disciplinary Academic Leaders}

Talented individuals should not only be equipped with rich TCM knowledge but also understand modern sciences and technologies and have an innovative spirit. Only these types of composite talents can become academic leaders and play a major role in the internationalization and development of TCM from China.

\subsubsection{Cultivating Composite Talents That Meet the Needs of TCM Internationalization}

We should try to cultivate professional TCM talents and composite talents who understand international quality standards, are familiar with TCM trade knowledge, are equipped with production and operation 
management capacities, understand research and development, know the requirements at home and abroad on the protection of intellectual property, keep a close watch on the dynamic progress of TCM scientific studies, and search for professional foreign language talents.

\subsection{Intellectual Property Strategy: Strengthen Intellectual Property Protection of TCM \\ 3.6.1. Enhancing the Awareness to Protect TCM Intellectual Property}

As intangible assets, intellectual property can effectively protect TCM inventions and creations. We must carry out intellectual property protection training among the practitioners of the TCM industry and enhance the awareness of the whole industry regarding the protection of intellectual property. We must learn the domestic and international regulations on the protection of intellectual property, which not only can protect the management performance of TCM enterprises but also can enhance their awareness of product protection. Therefore, in the internationalization of TCM, we must strengthen the protection of intellectual property and realize the purposes and significance of internationalization.

\subsubsection{Stepping Up Protection Efforts for TCM Intellectual Property}

We must strengthen the efforts to protect TCM intellectual property. Based on the current situation in China, we can make efforts in four areas. The first is to establish fast review and approval channels for TCM invention patents, accelerate the review and approval process and reduce the time needed. The second is to accelerate the handling of patent infringement lawsuit cases and significantly increase the amount of compensation to avoid wilful infringement. The third is to establish an arbitration institution for the TCM industry in order to reduce patent disputes. Fourth, we may also achieve effective protection through the core technologies, trademarks, essence and works in TCM intellectual property.

\section{References}

Balunas, M.J. and A.D. Kinghorn, 2005. Drug discovery from medicinal plants. Life Sciences, 78(5): 431-441. View at Google Scholar $\mid$ View at

Publisher
Liang, Y.Z., P. Xie and K. Chan, 2004. Quality control of herbal medicines. Journal of Chromatography B Analytical Technologies in the Biomedical \& Life Sciences, 812(1-2): 53-70. View at Google Scholar

Martins, E., 2014. The growing use of herbal medicines: Issues relating to adverse reactions and challenges in monitoring safety. Frontiers in Pharmacology, 4(4): 177. View at Google Scholar

Normile, D., 2003. The new face of traditional Chinese medicine. Science, 299(5604): 188-190. View at Google Scholar

Van, D.G.J., W.H. Van, J. Schroën, M. Wang, T. Hankemeier and G. Xu, 2010. Systems biology-based diagnostic principles as pillars of the bridge between Chinese and Western medicine. Planta Medica, 76(17): 2036-2047. View at Google Scholar | View at Publisher

Wagner, H., R. Bauer and D. Melchart, 2016. New analytical monographs on tcm herbal drugs for quality proof. Forschende Komplementarmedizin, 23 (Suppl 2): 16-20. View at Google Scholar $\mid$ View at Publisher

Wang, M., R.J.A.N. Lamers, H.A.A.J. Korthout, J.H.J.V. Nesselrooij, R.F. Witkamp and R.V.D. Heijden, 2005. Metabolomics in the context of systems biology: Bridging traditional chinese medicine and molecular pharmacology. Phytotherapy Research, 19(3): $173-182$. View at Google Scholar | View at Publisher

Ya, Y., X. Han, Y. Xie, Y. Wang and A. Lu, 2011. Analysis and thinking on the current status of traditional Chinese medicine standardization. World Science \& Technology, 13(3): 445-449. View at Google Scholar $\mid$ View at Publisher 